\title{
Delivering on the promise: HPV vaccines and cervical cancer
}

\section{John T. Schiller and Philip Davies}

Human papillomavirus (HPV) vaccines for the prevention of cervical cancer have produced encouraging results in recent clinical trials, and expectations are high that one or more vaccines will be licensed for commercial distribution within the next five years. The availability of an HPV vaccine would raise several implementation issues that must be addressed if the vaccine is to achieve the coverage necessary to significantly reduce the incidence of cervical cancer. The main implementation issues will differ between developing countries, where cervical cancer is often a leading cause of cancer deaths in women, and developed countries, where cervical cancer screening programmes have already substantially reduced the number of deaths from cervical cancer.

Vaccines that prevent viral diseases, such as polio, measles and smallpox, are among the most successful public health measures ever devised $^{1}$. The fact that cervical cancer is caused by a viral infection provides an exceptional opportunity to use vaccination as a tool for cancer prevention. The past two decades have seen the accumulation of extremely strong and consistent evidence that human papillomavirus (HPV) infections are a necessary cause of cervical cancer ${ }^{2}$ (TIMELINE). More than $99 \%$ of cervical cancers contain one or more of the approximately 15 HPV genotypes that have been associated with the development of cervical cancer. As approximately $50-60 \%$ of these cancers contain HPV16, and another 10-20\% contain HPV18 (REF. 3), these two HPV genotypes have been the focus of most vaccine development efforts. The same HPV genotypes are also involved in several other cancers, including vulvar, anal and oropharyngeal malignancies ${ }^{4}$, but the incidences of these cancers and the fractions that are attributable to HPV infection are lower, and so HPV vaccines have principally been used to target cervical cancer.

For many years, it was difficult to develop practical papillomavirus vaccines because these viruses do not grow efficiently in cultured cells ${ }^{5}$. Even if they did, live attenuated vaccines would contain viral oncogenes, which would probably prohibit their use as a prophylactic vaccine in healthy individuals. Attention therefore turned to the development of subunit vaccines based on the L1 major capsid protein. Early attempts to produce these in bacteria were unsuccessful because the purified proteins were predominantly malformed and unable to induce strong neutralizing antibody responses in animal models ${ }^{6}$. The breakthrough came with the finding that the L1 proteins could fold correctly and self-assemble into virus-like particles (VLPs) when expressed in eukaryotic cells. These VLPs not only closely resemble native virions morphologically (FIG. 1), but also induce high titres of antibodies that can prevent infection by authentic

\section{Timeline | HPV VLP vaccine development}

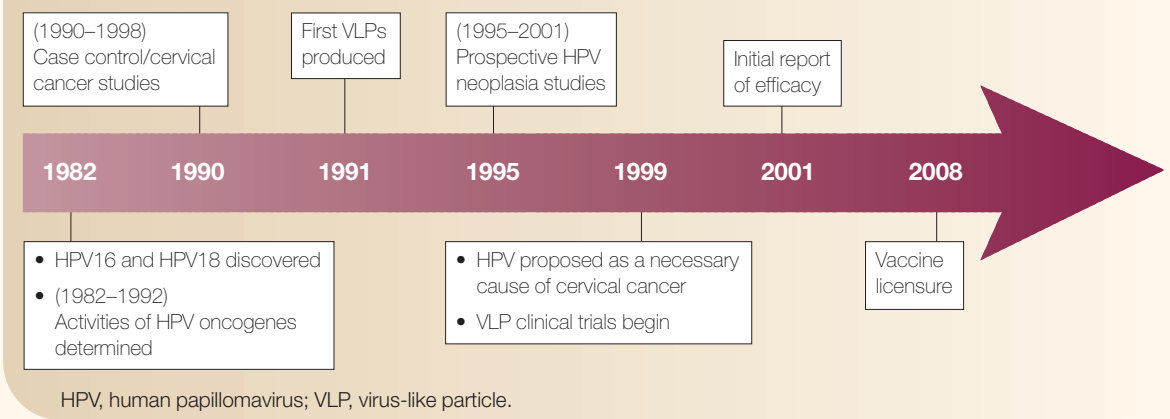

virions $^{7-9}$. However, as $L 1$ is the only viral gene required for vaccine production, the vaccines that are based on VLPs have no oncogenic potential.

Several clinical trials of HPV VLP-based vaccines have been conducted over the past five years, using VLPs that have been produced in either recombinant yeast- or baculovirusinfected insect cells ${ }^{10-14}$. VLPs are highly immunogenic in the 10-50 microgram dose range after three intramuscular injections, either with or without alum-based adjuvant, and the adverse side effects of vaccination have been minimal. A recent Merck-sponsored randomized and placebo-controlled proofof-concept efficacy trial using an HPV16 L1 VLP with alum vaccine in 1,500 young women reported a $99.7 \%$ seroconversion rate $^{15}$. After 1.5 years of follow-up, the vaccine was $100 \%$ effective at preventing persistent HPV16 infection and HPV16-induced cervical dysplasias (an early stage in cancer progression that is characterized by cell proliferation and disarray). The primary mediators of protection are thought to be virus-neutralizing antibodies, transudated from the serum into the cervical mucus. The same number of vaccinated and control patients developed cervical dysplasias containing other HPV types, supporting earlier in vitro virusneutralization studies that predicted protection by the VLP-based vaccines would predominantly be type-specific.

Large-scale efficacy trials of VLP vaccines are now being initiated by Merck, GlaxoSmithKline and the National Cancer Institute (TABLE 1). These trials have typespecific protection from the development of moderate and high-grade cervical dysplasias after incident HPV infection as their primary endpoint, in keeping with a US Food and Drug Administration (FDA) Advisory Committee recommendation for licensure. It is likely that these trials will be completed within the next five years. Although questions remain regarding the duration of protection and the effects of the menstrual cycle on protection, the consistently positive results so far have generated widespread optimism that a licensed HPV VLP vaccine will be made available to the public shortly after the trials are concluded. This prospect raises several important and complex implementation issues that must be addressed as a matter of urgency.

\section{Defining the target populations}

The HPV infections that cause cervical cancer are sexually transmitted, and, owing to their high prevalence, are generally acquired shortly after women become sexually active ${ }^{16,17}$. The expectations are therefore that an HPV VLP 


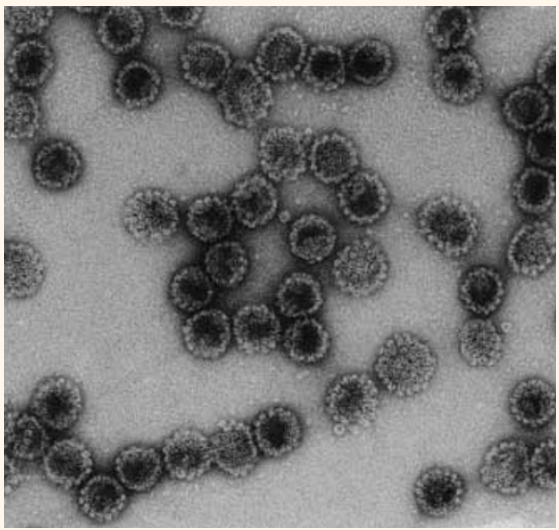

Figure 1 | Electronphotomicrograph of human papillomavirus (HPV)16 L1 virus-like particles. The virus-like particles were purified from L1recombinant baculovirus-infected insect cells. Staining is with uranyl acetate. Reproduced with permission from REF. 43 (C) (2000) Elsevier Science.

prophylactic vaccination programme will be most effective if it targets adolescent or pre-adolescent girls before the commencement of sexual activity. Implementing such a vaccination programme will be difficult as, at present, there are no public health intervention measures that routinely bring preadolescent girls to a clinic three times over a six-month period. Coverage would probably be increased if the vaccine could be administered as part of the infant immunization programme, perhaps with a booster injection at age 12 . However, a trial to investigate the effectiveness of this strategy would be daunting because it would take 15-20 years to complete. It is difficult to imagine a vaccine manufacturer sponsoring such a trial, and there seem to be no plans for a public-sector trial of this type.

The vaccination of sexually active women could prevent HPV infection in those who have not yet been exposed to the HPV genotypes contained in the vaccine. Unfortunately, VLP vaccination is unlikely to induce regression of established cervical dysplasias because the L1 protein is not expressed in the basal cells of infected epithelium where virus infection is thought to be maintained ${ }^{18}$. This conjecture is supported by studies in which VLP vaccination did not induce regression of papillomavirus-induced neoplasia (proliferative lesions) in animal models ${ }^{19}$. However, there are two potential benefits of vaccinating a woman currently infected with HPV16 or HPV18. The first is that it could decrease her risk of developing cervical cancer. Oncogenic HPVs infect many sites on the vulva, vagina and cervix ${ }^{17}$, but cancers usually develop at a metaplastic transition zone between the squamous and columnar epithelia in the cervix (known as the transformation zone; FIG.2 $)^{20}$. Vaccineinduced neutralizing antibodies that are present in the mucus of a woman's genital tract $^{21}$ might prevent the virus spreading from sites with low potential for malignant progression to the transformation zone. Second, vaccination of a woman with an active infection might decrease the likelihood of transmission to a new sexual partner because any shed virus could be inactivated by the neutralizing antibodies that are present in mucosal secretions. Although technically challenging, it would be desirable to determine the extent to which vaccination decreases the potential for transmission, as this would help to estimate the potential increase in herd immunity that could be achieved by vaccinating sexually active women. Given our current knowledge, it might be worthwhile to consider vaccinating sexually active young women in situations where it does not place an undue burden on healthcare resources. In situations where resources are more limited, it would probably be preferable to focus efforts on vaccinating pre-adolescents.

The vaccination of males is the subject of much debate. One would expect that vaccinating males against a sexually transmitted infection would decrease infection rates in women through herd immunity. However, in this instance, it is difficult to estimate the amount of protection vaccination would afford because the transmission dynamics of genital HPV infections are not well understood $^{22}$. Also, it is possible that the promising efficacy results seen in women might not be matched in men. The principal sites for oncogenic HPV infections in men are probably on the external genitalia, which would not be bathed in mucus and therefore would not be exposed to neutralizing antibodies after vaccination. Men could be protected if infections occur primarily at sites of trauma that result in the exudation of serum antibodies. However, the recent results of a herpes simplex glycoprotein $\mathrm{D}$ vaccine trial $^{23}$, reporting protection in women but not men, supports the possibility that the subunit HPV vaccine could have different efficacies in the two sexes. It is also likely that vaccination of men with prevalent infections will do little to inhibit the spread of infection, because virus shed from a man's external genital lesions would not normally contact his vaccine-induced neutralizing antibodies. The Merck vaccine, but not the GlaxoSmithKline vaccine, also contains HPV6 and HPV11 VLPs. As most genital warts are caused by these two genotypes, this vaccine has the potential to be licensed to

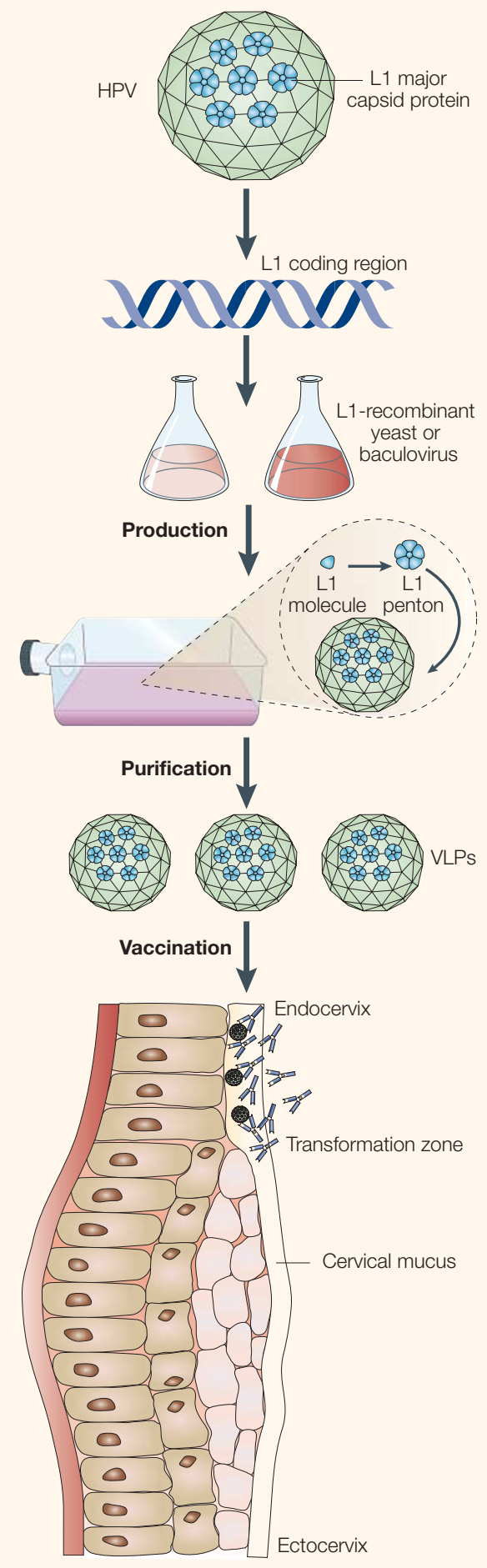

Figure 2 | Schematic representation of the production of, and vaccination with, human papillomavirus (HPV) virus-like particles

(VLPs). The HPV major capsid protein L1 can fold correctly and self-assemble into VLPS when expressed in eukaryotic cells. VLPs aim to protect against the development of cervical cancer; protection would be mediated by the induction of high titres of neutralizing antibodies against the HPV genotypes in the vaccine that prevent the virus infecting the transformation zone, a metaplastic area between the squamous and columnar epithelia in the cervix, where most cancers arise. 
treat this disease in men, providing efficacy is demonstrated. All trials so far have involved women. It will be important to conduct trials that specifically evaluate protection from genital HPV infection in men before making general decisions on whether to promote their vaccination. However, the lack of a wellvalidated procedure for sample collection to assess genital HPV infection in men could complicate the interpretation of the trial results.

\section{Public acceptance of HPV vaccination}

As HPV is a sexually transmitted infection, it is potentially subject to the same stigma that accompanies more widely recognized sexually transmitted infections, such as syphilis, chlamydia and HIV. It is therefore uncertain whether many parents would willingly accept vaccination of their eleven- or twelve-year-old daughters for sexually transmitted infections - they might perhaps prefer to think that this is an issue that could be addressed at a later time. The issue is further complicated because most of the general public have no knowledge of HPV, its involvement with cervical cancer or the need to vaccinate before exposure to the virus, and therefore have no basis on which to judge the importance or timing of HPV vaccination ${ }^{24}$. Acceptance could be increased by emphasizing the anticancer aspects of the vaccine, but it would be difficult and deceptive to ignore the connection to sexual transmission.

Overcoming the barriers to acceptance will require a coordinated public health education programme with clear information that achieves an appropriate balance between the sexual transmission and cervical carcinogenesis messages. If the messages are to be accepted by the target communities, it is also important to be sensitive to societal concerns about sexual activity in adolescents. The possibility has been raised that the vaccine could promote promiscuity, by relieving the fear of acquiring a cancer-causing sexually transmitted infection. Although it might seem unlikely that fear of HPV infection is a significant factor in decisions regarding sexual activity, public perceptions that the vaccine could promote an increase in sexual activity, or other similar concerns, are issues that must be evaluated and addressed.

It is also important to recognize that public health education programmes are complex undertakings. The messages that are associated with these programmes must be consistent, using terminology that is appropriate to the target audiences, and they must be communicated through several different channels. As such, they are best developed by people skilled in public health education, and should be coordinated at least at a national level, and

\begin{tabular}{|c|c|c|c|}
\hline Vaccine sponsor & HPV genotypes & Adjuvant & Trial sites \\
\hline National Cancer Institute & HPV16, HPV18 & ASO4 & Costa Rica \\
\hline GlaxoSmithKline & HPV16, HPV18 & AS04 & $\begin{array}{l}\text { United States, South } \\
\text { America, Europe }\end{array}$ \\
\hline Merck & HPV16, HPV18, HPV6, HPV11 & Alum & $\begin{array}{l}\text { United States, South } \\
\text { America, Europe }\end{array}$ \\
\hline
\end{tabular}

Thousands of young women will be followed for several years. Endpoints: CIN (cervical intraepithelial neoplasia) 2-3, 4-5 years follow-up; persistent HPV DNA, 2-3 years follow-up.

possibly higher, if there is a large degree of information exchange between neighbouring countries. Furthermore, these programmes take many years to have an effect. The prospects of achieving a noticeable impact on the public acceptance of HPV vaccination within the period of time that is envisaged for the introduction of the vaccine will be greatly reduced unless serious efforts are started immediately.

\section{Impact on cervical cancer screening} Cervical cancer is unusual in that highly effective screening programmes, based on cervical cytology (Pap) tests, already exist in many developed countries. In these countries, HPV vaccines must be considered both in light of their expected added value to cervical cancer prevention and their potential impact on compliance with cervical cancer screening. In countries such as the United States, which have high-quality, frequent and widespread screening programmes, the incidence of cervical cancers has decreased more than $80 \%$ since screening was first implemented (see Screening for cervical cancer in the online links box). It is very unlikely that programmes based on the current vaccine candidates could replace cervical cancer screening in these settings. Even overly optimistic projections of $100 \%$ coverage with a vaccine that is $100 \%$ effective against HPV16 and HPV18 would result in a vaccination programme that would be less effective than the best Pap screening programmes because at least $25 \%$ of cancers are caused by HPV genotypes other than HPV16 and HPV18.

However, cervical screening programmes are very expensive, costing an estimated US $\$ 6$ billion annually in the United States alone $^{25}$. Projections have been made that a combination of vaccination and screening might be more cost effective, with no compromise in protection, if the age at which patients are first screened was raised and/or screening intervals were increased ${ }^{26}$. It is noteworthy that these projections are sensitive to the cost of the vaccine and length of protection, two unknown variables at present. An effective vaccine would provide a benefit in terms of morbidity and cost by reducing the number of women with abnormal Pap tests, thereby reducing the number who must undergo colposcopic examination (an examination of the vagina and cervix using an instrument fitted with a low-power microscope), biopsy and surgical removal of premalignant lesions ${ }^{27}$. Finally, vaccination could also complement screening programmes by reaching those women who do not regularly attend. In countries with cervical screening programmes, approximately $50 \%$ of cervical cancers occur in women who do not attend for screening or are not screened regularly ${ }^{28}$. If these women could be reached by a vaccination programme, the public health benefits would be substantial.

There are concerns that widespread cervical cancer vaccination could lead to decreased compliance with Pap screening programmes. Women might erroneously assume that the vaccine provides protection against all cervical cancers, not just those caused by incident HPV 16 and HPV 18 infection. They might no longer feel the need to attend for screening, which they could find inconvenient or objectionable. If this occurred, then the introduction of an HPV vaccination programme could paradoxically lead to an increase in the incidence of cervical cancer. It could also lead to an increase in vulvar and perianal cancers, which would otherwise be diagnosed during the screening examinations. More generally, Pap screening often provides an important portal of entry for women into the healthcare system, and so the overall health of women could suffer if they abandon screening programmes. This trend could be countered by educating vaccinated women, but the issue of who will pay for the education programmes remains. The pharmaceutical companies can certainly be expected to educate physicians and the public on the benefits of vaccination. It is less clear to what extent they can be held responsible for promoting the continued desirability of cervical cancer screening.

\section{Vaccination in developing countries}

Cervical cancer is the leading cause of cancer deaths in women in many developing countries that lack organized screening programmes ${ }^{29}$, 


\begin{tabular}{|c|c|c|c|}
\hline Strategy & Potential advantage & Trials & References \\
\hline VLP aerosol administration & Ease of distribution & Animal and human* & 34 \\
\hline VLP plant production & Low production costs & Animal & 36,37 \\
\hline L2 minor capsid protein & Type cross-protective antibodies & Animal and human & 38,39 \\
\hline L1 recombinant Salmonella spp. & Low production costs/ease of distribution & Animal & 40 \\
\hline
\end{tabular}

*Unpublished meeting reports. HPV, human papillomavirus; VLP, virus-like particle.

and these countries could benefit enormously from an effective HPV vaccine. Models to estimate the relative benefits of HPV vaccination versus screening programmes for the cost-effective reduction in cervical cancer deaths in specific settings could be helpful to decision makers. However, these analyses are difficult at present, owing to the uncertainties concerning the cost, effectiveness and duration of protection for the vaccine, and the rapid development of alternative cervical cancer screening strategies ${ }^{30-32}$. One very important consideration is that effective screening programmes will have a rapid impact on the reduction of cervical cancer morbidity and mortality. Prophylactic vaccine programmes that target adolescents would take more than a decade to have an impact, as it generally takes at least this long for an incident HPV infection to progress to cancer. Even if vaccination was the most costeffective alternative, it might prove difficult for public health officials to champion a strategy that did not substantially reduce cervical cancer rates in the current generation of women.

The implementation of vaccination in developing countries is also complex. The experience with the hepatitis $B$ vaccine illustrates the difficulties of implementing a subunit anticancer vaccine in these settings. Twenty years after their introduction, hepatitis B immunization programmes have been introduced into only 130 of 206 countries, despite the current cost of just US $\$ 0.30$ per dose and a World Health Organization recommendation that it be incorporated into routine infant immunization programmes ${ }^{33}$. Several initiatives could be undertaken to shorten the timeline for an HPV vaccine. The first would be to undertake modest-scale proof-of-efficacy trials of the current vaccine candidates in countries of intermediate development with substantial cervical cancer burdens. In these countries, this could promote acceptance of - and advocacy for - HPV vaccines among both healthcare professionals and the public, which could increase uptake.
A second initiative would be to develop HPV vaccines that are better suited for distribution in low-resource settings. The current candidates are expensive to manufacture, as they require different VLPs for each HPV genotype that is covered in the vaccine, and the VLPs must be extensively purified from cultured eukaryotic cells. The vaccines will also be expensive to distribute, as they would involve a cold chain and three intramuscular injections of adolescents. Effective vaccines that could be manufactured less expensively, would not require a cold chain, could be administered without a needle (preferably in a single dose) and/or could prevent infection by many oncogenic genotypes would facilitate the introduction of vaccination programmes in low-resource settings and thereby increase the number of women that are protected from cervical cancer. It would also be desirable to develop a combined prophylactic/ therapeutic vaccine that could both prevent infection and induce regression of premalignant HPV-induced cervical lesions. This could decrease the incidence of cervical cancer in the current generation of women, while providing maximum protection for the next generation. A combined vaccine would also promote mass immunization campaigns, which could potentially lead to more rapid development of effective herd immunity. Some promising strategies that could potentially address one or more of the deficits of the current vaccine are currently under investigation (TABLE 2), but none is ready for large-scale clinical trials.

A crucial question for the development of second-generation vaccines is who will sponsor it. The prospect of two major vaccine manufacturers marketing an HPV vaccine in developed countries within a few years will almost certainly dampen enthusiasm for alternative vaccine development in the commercial sector. It therefore seems likely that it would have to be a predominately publicsector initiative, based on the support of governments and private foundations. Efforts are needed to convince these organizations that such an initiative is worthy of their support. It would not be a satisfactory outcome if HPV vaccines are proven to be safe and effective at preventing cervical cancer but are not made available to the women of the world who are most in need of them. John T. Schiller is at the National Cancer Institute,
NIH, Bethesda, Maryland, USA.

Philip Davies is at the European Cervical Cancer Association, Lyon, France.

Correspondence to J.T.S. e-mail:schillej@dc37a.nci.nih.gov

doi:10.1038/nrmicro867

1. Ehreth, J. The value of vaccination: a global perspective. Vaccine 21, 4105-4117 (2003).

2. Bosch, F. X., Lorincz, A., Munoz, N., Meijer, C. J. \& Shah, K. V. The causal relation between human papillomavirus and cervical cancer. J. Clin. Pathol. 55, 244-265 (2002).

3. Walboomers, J. M. et al. Human papillomavirus is a necessary cause of invasive cervical cancer worldwide. J. Pathol. 189, 12-19 (1999).

4. Gillison, M. L. \& Shah, K. V. Role of mucosal human papillomavirus in nongenital cancers. J. Natl Cancer Inst. Monogr. 31, 57-65 (2003).

5. Hagensee, M. \& Galloway, D. Growing human papillomaviruses and virus-like particles in the laboratory. Papillomavirus Report 4, 121-124 (1993).

6. Pilacinski, W. P. et al. in Papillomaviruses: Ciba Foundation Symposium 120, 136-156 (Wiley, UK, 1986)

7. Kirnbauer, R., Booy, F., Cheng, N., Lowy, D. R. \& Schiller, J. T. Papillomavirus L1 major capsid protein self-assembles into virus-like particles that are highly immunogenic. Proc. Natl Acad. Sci. USA 89 12180-12184 (1992)

8. Suzich, J. A. et al. Systemic immunization with papillomavirus $L 1$ protein completely prevents the development of viral mucosal papillomas. Proc. Nat Acad. Sci. USA 92, 11553-11557 (1995).

9. Breitburd, F. et al. Immunization with virus-like particles from cottontail rabbit papillomavirus (CRPV) can protect against experimental CRPV infection. J. Virol. 69, 3959-3963 (1995).

10. Zhang, L. F. et al. HPV6b virus-like particles are potent immunogens without adjuvant in man. Vaccine $\mathbf{1 8 ,}$ 1051-1058 (2000).

11. Harro, C. D. et al. Safety and immunogenicity trial in adult volunteers of a human papillomavirus $16 \mathrm{~L} 1$ virus-like particle vaccine. J. Natl Cancer Inst. 93, 284-292 (2001).

12. Evans, T. G. et al. A Phase 1 study of a recombinant virus-like particle vaccine against human papillomavirus type 11 in healthy adult volunteers. J. Infect. Dis. 183, 1485-1493 (2001).

13. Brown, D. R. et al. Neutralization of human papillomavirus type 11 (HPV-11) by serum from women vaccinated with yeast-derived HPV-11 L1 virus-like particles: correlation with competitive radioimmunoassay titer. J. Infect. Dis. 184, 1183-1186 (2001).

14. Emeny, R. T. et al. Priming of human papillomavirus type 11-specific humoral and cellular immune responses in college-aged women with a virus-like particle vaccine. J. Virol. 76, 7832-7842 (2002).

15. Koutsky, L. A. et al. A controlled trial of a human papillomavirus type 16 vaccine. N. Engl. J. Med. $\mathbf{3 4 7}$, 1645-1651 (2002). 
16. Burk, R. D. et al. Declining prevalence of cervicovaginal human papillomavirus infection with age is independent of other risk factors. Sex. Transm. Dis. 23, 333-341 (1996)

17. Winer, R. L. et al. Genital human papillomavirus infection: incidence and risk factors in a cohort of female university students. Am. J. Epidemiol. 157, 218-226 (2003).

18. Stubenrauch, F. \& Laimins, L. A. Human papillomavirus life cycle: active and latent phases. Semin. Cancer Biol. 9. 379-386 (1999).

19. Kirnbauer, R. et al. Virus-like particles of bovine papillomavirus type 4 in prophylactic and therapeutic immunization. Virology 219, 37-44 (1996).

20. Eifel, P. J., Berek, J. T. \& Thigpen, J. T. in Cancer Principles \& Practice of Oncology 6th Ed. (eds DeVita, V. T. Hellman, S. \& Rosenberg, S. A.) 1526-1572 (Lippincott, Williams and Wilkins, Philadelphia, 2001).

21. Nardelli-Haefliger, D. et al. Specific antibody levels at the cervix during the menstrual cycle of women vaccinated with human papillomavirus 16 virus-like particles. J. Natl Cancer Inst. 95, 1128-1137 (2003).

22. Hughes, J. P., Garnett, G. P. \& Koutsky, L. The theoretical population-level impact of a prophylactic human papilloma virus vaccine. Epidemiology 13, 631-639 (2002).

23. Stanberry, L. R. et al. Glycoprotein-D-adjuvant vaccine to prevent genital herpes. N. Engl. J. Med. 347, 1652-1661 (2002)

24. Waller, J. et al. Awareness of human papillomavirus among women attending a well woman clinic. Sex. Transm. Infect. 79, 320-322 (2003).

25. Kurman, R. J., Henson, D. E., Herbst, A. L., Noller, K. L. \& Schiffman, M. H. Interim guidelines for management of abnormal cervical cytology. The 1992 National Cancer Institute workshop. JAMA 271, 1866-1869 (1994).

26. Kulasingam, S. L. \& Myers, E. R. Potential health and economic impact of adding a human papillomavirus vaccine to screening programs. JAMA $290,781-789$ (2003)

27. Goldie, S. J. et al. A comprehensive natural history model of HPV infection and cervical cancer to estimate the clinical impact of a prophylactic HPV-16/18 vaccine. Int. J. Cancer 106, 896-904 (2003).

28. Sasieni, P. D., Cuzick, J. \& Lynch-Farmery, E. Estimating the efficacy of screening by auditing smear histories of women with and without cervical cancer. Br. J. Cancer 73, 1001-1005 (1996).
29. Parkin, D. M., Bray, F., Ferlay, J. \& Pisani, P. Estimating the world cancer burden: Globocan 2000. Int. J. Cance 94, 153-156 (2001).

30. Wright, T. C. Jr, Denny, L., Kuhn, L. \& Goldie, S. Use of visual screening methods for cervical cancer screening. Obstet. Gynecol. Clin. North Am. 29, 701-734 (2002)

31. Franco, E. L. Primary screening of cervical cancer with human papillomavirus tests. J. Natl Cancer Inst. Monogr. 31, 89-96 (2003).

32. Kulasingam, S. L. \& Koutsky, L. A. Will new human papillomavirus diagnostics improve cervical cancer control efforts? Curr. Infect. Dis. Rep. 3, 169-182 (2001).

33. Kao, J. H. \& Chen, D. S. Global control of hepatitis B virus infection. Lancet Infect. Dis. 2, 395-403 (2002).

34. Balmelli, C., Demotz, S., Acha-Orbea, H., De Grandi, P. \& Nardelli-Haefliger, D. Trachea, lung, and tracheobronchial lymph nodes are the major sites where antigen-presenting cells are detected after nasal vaccination of mice with human papillomavirus type 16 virus-like particles. J. Virol. 76, 12596-12602 (2002).

35. Rose, R. C. et al. Oral vaccination of mice with human papillomavirus virus-like particles induces systemic virus-neutralizing antibodies. Vaccine 17, 2129-2135 (1999).

36. Biemelt, S., Sonnewald, U., Galmbacher, P., Willmitzer, L. \& Muller, M. Production of human papillomavirus type 16 virus-like particles in transgenic plants. J. Virol. 77, 9211-9220 (2003)

37. Warzecha, $\mathrm{H}$. et al. Oral immunogenicity of human papillomavirus-like particles expressed in potato. J. Virol. 77, 8702-8711 (2003).

38. Kawana, K. et al. Safety and immunogenicity of a peptide containing the cross-neutralization epitope of HPV16 L2 administered nasally in healthy volunteers. Vaccine $\mathbf{2 1}$, 4256-4260 (2003).

39. Roden, R. B. et al. Minor capsid protein of human genital papillomaviruses contains subdominant, crossneutralizing epitopes. Virology 270, 254-257 (2000).

40. Nardelli-Haefliger, D. et al. Human papillomavirus type 16 virus-like particles expressed in attenuated Salmonella typhimurium elicit mucosal and systemic neutralizing antibodies in mice. Infect. Immun. $\mathbf{6 5}$ 3328-3336 (1997)

41. Muller, M. et al. Chimeric papillomavirus-like particles. Virology 234, 93-111 (1997).
42. Greenstone, H. L. et al. Chimeric papillomavirus virus-like particles elicit antitumor immunity against the $\mathrm{E} 7$ oncoprotein in an HPV16 tumor model. Proc. Natl Acad. Sci. USA 95, 1800-1805 (1998).

43. Schiller, J. T. \& Hidesheim, A. Developing HPV virus-like particle vaccines to prevent cervical cancer: a progress report. J. Clin. Virol. 19, 67-74 (2000).

\section{Acknowledgements}

We thank D. Lowy and M. Allende for their helpful comments during the preparation of the manuscript.

\section{Disclaimer}

The views expressed in this article are those of the authors and do not represent the official positions of their respective organizations.

Competing interests statement

The authors declare competing financial interests; see Web version for details.

\section{(4) Online links}

\section{DATABASES}

The following terms in this article are linked online to: Entrez: http://www.ncbi.nlm.nih.gov/Entrez/

HPV16 | HPV18

Infectious Disease Information:

http://www.cdc.gov/ncidod/diseases/index.htm

chlamydia | hepatitis B | HIV | syphilis

SwissProt: http://www.ca.expasy.org/sprot/

L1 major capsid protein

\section{FURTHER INFORMATION}

Cervical cancer:

http://www.nlm.nih.gov/medlineplus/cervicalcancer.html European Consortium for Cervical Cancer Education: http://www.eccce-cervical-cancer.org/contents/home/index.asp/ NHS cervical screening programme:

http://www.cancerscreening.nhs.uk/cervical/ Screening for cervical cancer:

http://cancer.gov/cancerinfo/pdq/screening/cervical/ healthprofessional

John T. Schiller's laboratory:

http://ccr.cancer.gov/staff/staff.asp? staffid $=443$

Access to this interactive links box is free online. 